\title{
How we managed total parenteral nutrition in our hospital in 2006-2007
}

\author{
M. Wilczynska, I. M. Patanwala and J. Sayer \\ Department of Gastroenterology, Doncaster Royal Infirmary, Armthorpe Road, Doncaster, DN2 5LT, UK
}

Total parenteral nutrition (TPN) is an important supportive therapy for all the malnourished or at risk of malnutrition patients who meet the criteria set out in the national guideline. ${ }^{(1)}$ We analyzed and compared data for TPN administration, collected in the selected periods within 2 years.

An audit in the 2006 resulted in the introduction of the proforma for TPN administration ${ }^{(2)}$. A reudit in the 2007 aimed to assess the requirement for further changes including the introduction of Nutritional Support Team (NST). Many international studies confirm that

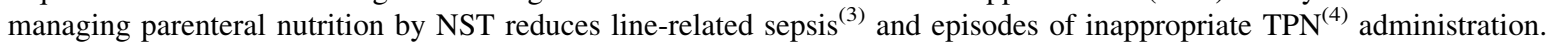

Data were collected retrospectively using a pharmacy-generated list of the all new TPN prescriptions. Thirty-one episodes of TPN administration were recorded between May and July 2007 and 53 episodes between January and May 2006. Information including demographics, duration and the person initiating TPN was collected for all cases (Table 1). A one-way analysis of variance test was used to look for statistical differences in the mean duration of TPN between two selected periods but there was no significant difference with a $P$ value of 0.867 .

Table 1. Demographics

\begin{tabular}{lll}
\hline & \multicolumn{1}{c}{2006} & \multicolumn{1}{c}{2007} \\
\hline Mean age & 62.3 years & 65.3 years \\
Gender & $40 \% \mathrm{~F}$ & $52 \% \mathrm{~F}$ \\
Location of TPN initiation & $70 \%$ High dependency care & $42 \%$ High dependency care \\
Mean duration of TPN & 10.47 days & 10.50 days \\
The person prescribing TPN & Anesthetists 50\%, surgeons $45 \%$ & Anesthetists 50\%, surgeons 50\% \\
\hline
\end{tabular}

In addition, the first 18 cases from each period were examined for: indications (graph 1), type of venous access used (graph 2), complications (Table 2), and reason for stopping parenteral nutrition.

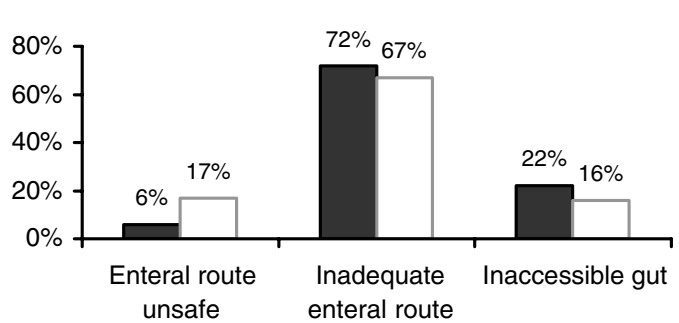

Graph 1. Indications for TPN use.

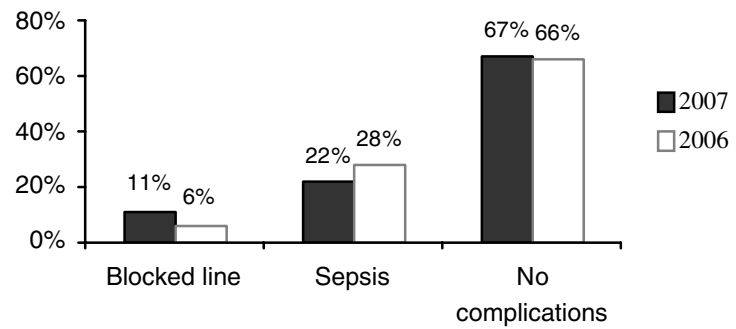

Graph 2. Complications were related to CVP line insertion.

Table 2. Venous access used for TPN administration

\begin{tabular}{ccccc}
\hline & PICC $(\%)$ & Hickman line $(\%)$ & Peripheral cannulae $(\%)$ & Central not tunneled line $(\%)$ \\
\hline 2006 & 5 & 17 & 11 & 67 \\
2007 & 6 & 11 & 22 & 61 \\
\hline
\end{tabular}

In $78 \%$ of the cases, TPN was stopped when the enteral route was established and the patients' nutritional needs were met by other means; there was no difference between investigated periods.

Comparing data from 2006 and 2007, there were no major improvements in the TPN use especially with regard to duration and episodes of central line-related sepsis (line culture and blood culture growing the same organism). In a view of the data from research and our audit, we agreed that it is important to introduce further changes including establishment of the NST.

1. NICE (2006) Clinical Guideline 32. Nutrition support in adults.

2. Patanwala IM \& Sayer JM (2008) Proceedings of the Nutrition Society. 2008 May; 67, OCE4, E104.

3. Kennedy JE \& Nightingale JM (2005) Nutrition 21, 1127-1133.

4. Olveira Fuster G, Mancha Doblas I, González-Romero S et al. (2000) Nutr Hosp 15, 118-122. 